\title{
Belief Update Methods and Rules-SOME COMPARISONS
}

\author{
LESZEK WROŃSKI \\ Jagiellonian University
}

\begin{abstract}
We tackle two open questions from Leitgeb and Pettigrew (2010b) regarding what the belief update framework described in that paper mandates as correct responses to two problems. One of them concerns credences in overlapping propositions and is known in the literature as the "simultaneous update problem". The other is the well known "Judy Benjamin" problem concerning conditional credences. We argue that our results concerning the problems point to deficiencies of the framework. More generally, we observe that the method of minimizing inverse relative entropy seems to work better than (or at least equally well as) its competitors in many situations.
\end{abstract}

S UPPOSE that an agent at a certain time has credences in propositions which form a Boolean algebra. These credences, or degrees of belief (we will use these terms interchangeably), are given by a "belief function" $b$. Suppose that subsequently the agent obtains evidence which entails constraints that $b$ does not meet. Which belief function should the agent adopt? This paper will be concerned with problems of this kind ("belief update problems"), and in particular with two specificic issues posed as open problems at the end of Leitgeb and Pettigrew (2010b).

Some belief update problems have answers with which many people feel comfortable; the most common example would be that if, for some proposition $E$, the agent learns that $E$ (and nothing more), she should update her belief function by conditionalization, provided that her initial credence in $E$ was not equal to zero (more on this in Section [1). However, with respect to other problems intuitions may vary wildly. What if the evidence entails (only) new credences in two overlapping propositions? (This is the "simultaneous update problem".) Or what if it entails (only) a new conditional credence? (This is the "Judy Benjamin problem".) Some might want to say that in such cases there is no unique answer; that is, that the evidence underdetermines the choice of the new belief function. Still, some answers may be more reasonable than others. Given a belief update

Contact: Leszek Wroński <leszek.wronski@uj.edu.pl> 
framework, it may be fruitful to check whether it suggests a specific reaction in such cases, and if so, whether the form of it may suggest some shortcomings of the framework we might want to try to avoid.

What follows requires some setting up. We assume that we are considering an agent who holds credences in a Boolean algebra of propositions which are subsets of a finite set $W$ which has $n$ elements. ${ }^{1}$ We can think of $W$ as containing worlds epistemically possible for the agent. Each $w_{i} \in W$ is to be identified with a unit vector in $\mathbb{R}^{n}$ (with 0 s on all axes apart from the $\mathrm{i}^{\text {th }}$ and a 1 on the $\mathrm{i}^{\text {th }}$ ). The credences are given by a function $b$. We assume we are dealing with probabilistic agents-that is, $b$ is a classical probability function. Thanks to this assumption, we can think of a belief function $b$ as an element of $\mathbb{R}^{n}$, namely, a tuple containing the values of $b\left(\left\{w_{i}\right\}\right)$ for all $i$. Throughout the paper $b$, if not treated as a variable, will always be the "prior" belief function of the considered agent, that is, the one she has before she receives the evidence which are the topic of the given belief update problem, and $b^{\prime}$ will be the function to be adopted after she receives that evidence. And so $b$ will be the "prior" belief function, and $b^{\prime}$ the "posterior" one.

The structure of the paper is as follows. In Section 1 I will propose a distinction between belief update methods and rules which I hope will be beneficial to the clarity of the arguments. Section 2 will cover the Judy Benjamin problem. After that, in Section 3 we will tackle the simultaneous update problem-the main results of the paper are there, with proofs given in the Appendix.

\section{Belief Update Methods and Rules}

One can propose a way of updating belief functions by formulating statements which may be varyingly specific. One can, for example, say "always, if possible, minimize relative entropy". This way, one has a pleasingly general proposal which may, on the other hand, be at first glance uninformative in specific cases: how does one go about obtaining the needed belief function in a given situation? On the other hand, one can say, for example, "when you learn that $E$ is true (and nothing more), adopt, if possible, the belief function $b^{\prime}$ such that for any proposition $A b^{\prime}(A)=b(A \mid E)^{\prime \prime}$. This way, one has an easy rule of constructing the new belief function provided the evidence is in a certain format. I will call statements of the first kind "methods", and those of the second kind "rules". Methods do not distinguish between the different types of constraints which are implied by evidence; rules do. Rules can be motivated by methods; in fact, once the appropriate definitions are given, one may attempt to prove theorems to the effect that a rule (say, Conditionalization-see below, p. 297) follows from a method (say, Minimizing Relative Entropy-see below, p. 296). If there are

1. We make the finitude assumption following Leitgeb and Pettigrew (2010a). 
convincing arguments against a rule which follows from a given method, then they speak also against that method. In this section I will give definitions of methods and rules which I will use later.

\subsection{Methods}

Suppose one subscribes to the popular project of accuracy-first epistemology ${ }^{2}$ and claims to have a specific inaccuracy measure $I\left(b, w_{i}\right)$ which associates with each belief function $b$ and possible world $w_{i}$ a number which, intuitively, is to be a measure of $b$ 's "distance from truth" if $w_{i}$ is the actual world. Then one could propose a belief update method by saying "always, if possible, minimize expected inaccuracy". The specifics will depend on the inaccuracy measure proposed and the chosen way of calculating its expected value. In the case of the framework from Leitgeb and Pettigrew (2010a), which we will henceforth dub the "LP" framework, and in the context of which the two problems mentioned above will first be considered, the so called "quadratic inaccuracy measures" are used. The relevant method is the following:

\section{The Quadratic Update Method (QUM):}

Given evidential constraints $C$, the belief function $b^{\prime}$ which should be adopted is such that it satisfies $C$ and minimizes the expression

$$
\sum_{n} b\left(\left\{w_{n}\right\}\right)\left\|w_{n}-b^{\prime}\right\|^{2}
$$

Remember that $b$ is the agent's belief function before she receives new evidential constraints, and that since we assume that belief functions are probability functions, $b^{\prime}$ can be considered as a vector. The "the" in the formulation of (QUM) is there because for all update problems discussed in this paper the empirical constraints define a convex and closed set of belief functions, so the appropriate minimum exists. This is the case for all update methods considered here.

After giving a critique of the LP framework, Levinstein (2012) proposes that the inaccuracy of a function $b$ if $w_{i}$ is the actual world be captured just by $-\ln b\left(\left\{w_{i}\right\}\right) .{ }^{3}$ This leads to the following method: (1998).

2. For a recent monograph see Pettigrew 2016. For the field-opening paper see Joyce

3. Those familiar with the formal epistemology literature should recognize this as using "the only local proper scoring rule", see e.g., Parry, Dawid, and Lauritzen 2012. 
The Local-logarithmic Update Method (LLM):

Given evidential constraints $C$, the belief function $b^{\prime}$ which should be adopted is such that it satisfies $C$ and minimizes the expression

$$
-\sum_{n} b\left(\left\{w_{n}\right\}\right) \cdot \ln b^{\prime}\left(\left\{w_{n}\right\}\right)
$$

Notice that both of these methods pay attention to the expected inaccuracy of a considered future belief function as calculated from the perspective of the current one, that is, one which the agent finds to be incompatible with the evidence. For doubts regarding the reasonability of this see, e.g., Pettigrew (2016: Chapter 15). If I were to mount a defense of this, I would attempt to say that it is only due to having the particular initial function that what the agent experiences is interpreted as this, and not some other, piece of evidence, for example learning that a particular proposition $E$ is true. Spelling this out properly would require a whole formal system modelling evidence which I am not able to give now.

The remaining two methods I would like to introduce here use the notion of relative entropy. The first one is widely used in epistemology, the second one less so.

\section{The Method of Minimizing Relative Entropy (MRE):}

Given evidential constraints $C$, the belief function $b^{\prime}$ which should be adopted is such that it satisfies $C$ and minimizes the expression

$$
\sum_{n} b^{\prime}\left(\left\{w_{n}\right\}\right) \ln \left(\frac{b^{\prime}\left(\left\{w_{n}\right\}\right)}{b\left(\left\{w_{n}\right\}\right)}\right) .
$$

\section{The Method of Minimizing Inverse Relative Entropy (MIRE):}

Given evidential constraints $C$, the belief function $b^{\prime}$ which should be adopted is such that it satisfies $C$ and minimizes the expression

$$
\sum_{n} b\left(\left\{w_{n}\right\}\right) \ln \left(\frac{b\left(\left\{w_{n}\right\}\right)}{b^{\prime}\left(\left\{w_{n}\right\}\right)}\right) .
$$

Notice that the only difference between the two expressions to be minimized is the position of the variable. In the MRE expression the variable, that is, the value of the "new" belief function $b^{\prime}$, occurs twice in each summand, while in the MIRE expression it does so only once, since $b$ and $b^{\prime}$ switch their roles. Readers who have encountered these expressions before might wonder about the choice of the labels; they might for example have arguments for the reverse labelling of the notions, referring to the first one above, and not the second, as minimizing 
inverse relative entropy. I do not want to enter into such debates; if one wants to label these notions in a different way, I will not oppose. I would not like to suggest any particular "directionality" here, so I will avoid writing that, say, MRE minimizes relative entropy "from" $b$ to $b^{\prime}$, or vice versa. For obvious reasons of (lack of) symmetry, I will also never write that what we are minimizing is the (inverse) relative entropy "between" $b$ and $b^{\prime}$. I believe that doing any of those two things can only cause confusion. ${ }^{4} \mathrm{I}$ am using the labels as they appear in the recent and influential paper Douven and Romeijn (2011). In the 1981 paper in which van Fraassen introduced the Judy Benjamin problem, he referred to the MRE method as "InfoMin", that of minimizing relative information.

The first small observation I would like to make in this paper is the following: the method of minimizing inverse relative entropy is equivalent to the locallogarithmic method. Simply, the relevant expressions share their minima. The MIRE expression looks similar to an expected value calculated from the perspective of the belief function $b$. This might be interesting for some who would like to link entropy with epistemic inaccuracy. I will not pursue this matter here.

\subsection{Rules}

Here I will introduce the belief update rules-that is, the ways of updating one's belief function in response to evidence of a specific type-which we will use later. The first one is the well known conditionalization rule.

\section{The Full Conditionalization Rule (FC):}

Evidence: entails (only) that a proposition $E$ is true.

Response: if $b(E)>0$, then $b^{\prime}$ should be such that for any proposition $A$ $b^{\prime}(A)=b(A \mid E)$.

It can be shown by a limit argument that the FC rule follows from both MRE and MIRE (see remarks in Chapter 15 of Pettigrew, 2016). How it relates to QUM is a more delicate matter to which we will turn soon. In what follows I will refer to the evidence which features in FC, namely, evidence which entails (only) that a proposition $E$ is true, by the name "Bayesian evidence".

Let us now present the rule which many think to be a generalization of FC:

4. For an example, see the "Talk" page of the Wikipedia entry for the Kullback-Leibler divergence, Section 12. 


\section{The Jeffrey Conditionalization Rule (JC):}

Evidence: entails (only) new credences $q_{1}, \ldots, q_{m}$ in propositions $E_{1}, \ldots, E_{m}$ which form a partition of $W$.

Response: if for any $i \in\{1, \ldots, m\} b\left(E_{i}\right)>0$, then $b^{\prime}$ should be such that for any proposition $A$

$$
b^{\prime}(A)=\sum_{i=1}^{m} q_{i} \cdot b\left(A \mid E_{i}\right)
$$

That the JC rule follows from both MRE and MIRE methods can again be shown by a limit argument (see Pettigrew 2016). For the cases in which all $q_{i}{ }^{\prime} \mathrm{s}$ are nonzero it was proven in Diaconis and Zabell (1982).

However, the quadratic method used in the LP framework entails a different response to the same information:

\section{The Alternative Jeffrey Conditionalization Rule (AJC):}

Evidence: entails (only) new credences $q_{1}, \ldots, q_{m}$ in propositions $E_{1}, \ldots, E_{m}$ which form a partition of $W$.

Response: For any $i \in\{1, \ldots, m\}$ let $d_{i}$ be the unique number such that

$$
\left(\sum_{\left\{w \in E_{i}: b(\{w\})+d_{i}>0\right\}} b(\{w\})+d_{i}\right)=q_{i} .
$$

Then $b^{\prime}$ should be such that for any $w \in W$ such that $w \in E_{i}$,

$$
b^{\prime}(\{w\})= \begin{cases}b(\{w\})+d_{i} & \text { if } b(\{w\})>0 \\ 0 ; & \text { otherwise. }\end{cases}
$$

In what follows, I will refer to the evidence which features in JC and AJC, namely, evidence which entails (only) new credences in propositions forming a partition of $W$, by the name "Jeffrey evidence".

Since we assume probabilism, AJC uniquely determines $b^{\prime}$. Notice that AJC is applicable in some cases in which JC is not, since the former does not need to assume that the initial credences in the $E_{i}$ 's are nonzero. Consult Leitgeb and Pettigrew (2010b: 255) for a geometric interpretation of AJC. The rule has been criticized by Levinstein (2012) for its various shortcomings. Among those not covered in that paper but discussed by Leitgeb and Pettigrew themselves is AJC's cardinality dependence: unlike in the case of JC, the new credence in an arbitrary proposition $A$ depends not only on the prior credences in the various $E_{i}$ 's and the conditional credences of the form $b\left(A \mid E_{i}\right)$, but also on how many elements the $E_{i}{ }^{\prime}$ 's have. 
You might justifiably think that AJC is incompatible with FC. It is easy to construct a $W$, a belief function $b$ and a proposition $E$ such that if we first assume that one's evidence entails that $b^{\prime}(E)=1$ and $b^{\prime}(\neg E)=0$ and then use AJC, we will end up with a different function $b^{\prime}$ from the one we would have reached had we initially assumed that the evidence entailed that one learned that $E$ and then used FC. However, in the LP framework both AJC and FC are valid. This is because the authors think that the two situations should be modelled differently. Namely, the authors think that learning that $E$ requires one to narrow one's set of epistemically possible worlds, while obtaining credence 1 in $E$ and credence 0 in $\neg E$ does not. This is just one of the subtleties which lead them to endorse something different from the QUM above in cases of one's learning that some proposition is true. ${ }^{5}$ This way they are able to obtain the surprising result that their framework endorses both AJC and FC. Fortunately, for all other belief update situations concerned in Leitgeb and Pettigrew (2010b) apart from "learning that $E$ ", and in particular for the situations which are at the heart of the two problems to which we will now turn, the authors decide on QUM as the belief update method. ${ }^{6}$

I will now turn to the two belief update problems posed in Leitgeb and Pettigrew (2010b: 262-263), adjusting the wording to the formalism used in the current paper. I will approach the problems in what I believe to be the order of increasing difficulty.

\section{The Judy Benjamin Problem}

The essence of the problem is the following: for some propositions $A$ and $B$, your evidence entails (only) your new conditional credence in $A$ given $B$. What should your new belief function look like?

Observe that JC cannot be used, at least not until the evidence is processed in some way which would lead to establishing new credences in all elements of some partition of $W$. We need, then, a different rule. Some people think there are many belief functions it would be rational to adopt in this situation; in other words, that no unique solution exists. A recent detailed exposition of such a view can be found in Huisman (2014). A (too) quick response would be that formal epistemology treats belief update problems in the context of ideal rationality, which may imply in every evidential situation a unique rational belief update procedure, even if we, real agents, can only attempt to imperfectly imitate this ideal. Another direction would be to notice that there are obviously ridiculous ways of responding to such evidence (say, "set $b^{\prime}(B)$ to $I^{\prime \prime}$ ), and some other res-

5. Another subtlety concerns the distinction between "local" and "global" inaccuracy; see Leitgeb, Hannes and Richard Pettigrew 2010a 204).

6. The authors call it "minimizing expected global inaccuracy". 
ponses which many people find to be unintuitive (more on that below). If one's update method prescribes some such response, this would be to its disadvantage. Here is the original illustration of the problem from the paper as introduced by van Fraassen (1981):

"[Judy Benjamin (a soldier)] and her patrol are dropped in a swampy area which they have to patrol. The area is divided into the region of the Blue Army, to which Judy Benjamin and her fellow soldiers belong, and that of the Red Army. The Red Army region is further divided into Headquarters Company Area and Second Company Area. The patrol has a map which none of them understands, and they are soon hopelessly lost. Using their radio they are at one point able to contact their own headquarters. After describing whatever they remember of their movements, they are told by the duty officer 'I don't know whether or not you have strayed into Red Army territory. But if you have, the probability is $3 / 4$ that you are in their Headquarters Company Area.' At this point the radio gives out." (van Fraassen 1981)

There seem to be three propositions of interest: $B$ ("JB's unit is in the Blue Army region"), $R_{1}$ ("JB's unit is in the Red Army region, Headquarters Company Area"), and $R_{2}$ ("... Red Army region, Second Company Area"). $B, R_{1}$ and $R_{2}$ are pairwise incompatible and jointly exhaustive; that is, they form a partition of whatever $W$ we use as the set of Judy's epistemically possible worlds. The evidence Judy receives seems to entail (only) that $b^{\prime}\left(R_{1}\right)=3 \cdot b^{\prime}\left(R_{2}\right) .{ }^{7}$ Her prior belief function $b$ is set to $b\left(R_{1}\right)=b\left(R_{2}\right)=0.25, b(B)=0.5$. Van Fraassen's (1981) discovery is that according to MRE the belief function $b^{\prime}$ which Judy should adopt is such that $b^{\prime}(B)=0.532$, approximately. (This holds regardless of the cardinality of $W$.) Many, including van Fraassen himself, found this result to be unintuitive: why should Judy's degree of belief that her unit is in the Blue Army region increase? What the duty officer says seems to concern only the Red Army areas. In fact, for any fraction different from 3/4 (and 1 or o) the situation is similar: MRE has it that Judy's credence in $B$ should increase. (See Lukits, 2014, for a recent defence of this view.) I will now argue that the LP framework shares this unintuitive feature due to employing QUM as its belief update method.

I already mentioned above that the updating rule prescribed by the LP framework in cases involving Jeffrey evidence displays dependence on cardinalities. It turns out that the same is true for the Judy Benjamin problem. Let us first consider the smallest possible case, with $W$ as $\left\{w_{1}, w_{2}, w_{3}\right\}, R_{1}=\left\{w_{1}\right\}, R_{2}=$ $\left\{w_{2}\right\}, B=\left\{w_{3}\right\}$, and of the initial belief function function $b$ as the triple $\langle 0.25$,

7. In this presentation we abstract away from details like the need to model Judy's beliefs in the reliability of her duty officer. For an approach exploring this direction see Grove and Halpern (1997). 
$0.25,0.5\rangle$. A short excercise in minimizing a real valued function of one variable shows that according to QUM the belief function $b^{\prime}$ that Judy should adopt is such that $b^{\prime}(B)=7 / 13$. And so, just like with MRE, the LP framework will have Judy increase her degree of belief in that her unit is in the Blue Army region. Still keeping with the 3-element $W$, we can generalize a bit with respect to the evidence given, and assume that the evidence entails, for some non-negative $m$, that $b^{\prime}\left(R_{1}\right)=m \cdot b^{\prime}\left(R_{2}\right){ }^{8}$ A slightly longer excercise in analysis ${ }^{9}$ shows that if $m$ is not equal to 1 , then according to QUM $b^{\prime}(B)>b(B)$. Therefore we can see that if we use the smallest possible (3 element) model for the situation then QUM performs similarly to MRE, that is, it shares with it the disadvantage of not giving the intuitive solution (which would keep the degree of belief in $B$ constant). I do not think the particular numeric details of the posterior credences are important.

A variant of the Judy Benjamin problem involves splitting the Blue Army region into two areas, mirroring those of the Red Army region, and assuming that Judy's initial belief function is uniform with regard to the placement of her unit in the four regions involved. Consider, then, the case in which $W=$ $\left\{w_{1}, w_{2}, w_{3}, w_{4}\right\}, b\left(\left\{w_{i}\right\}\right)=1 / 4$ for any $i \in\{1, \ldots, 4\}, R_{1}=\left\{w_{1}\right\}, R_{2}=\left\{w_{2}\right\}, B_{1}=$ $\left\{w_{3}\right\}, B_{2}=\left\{w_{4}\right\}$, and $B=B_{1} \cup B_{2}$. If, as above, the evidence entails (only) that $b^{\prime}\left(R_{1}\right)=3 \cdot b^{\prime}\left(R_{2}\right)$, then QUM dictates that $b^{\prime}(B)=5 / 9$. And so according to the LP framework Judy's posterior credence in $B$ depends on the cardinality of the propositions involved..$^{10}$ I do not want to claim that this is a particular problem for the framework, since I believe the points from the previous paragraph already show that its belief update method, QUM, does not fare better than MRE in response to the Judy Benjamin problem.

Consider, however, what LLM would mandate as the solution here. Take the original formulation of the problem and start with the simplest case with $W$ having 3 elements. The prior belief function $b$ is, again, $\langle 0.25,0.25,0.5\rangle$. The posterior belief function $b^{\prime}$ can be written as $\langle 3 \beta, \beta, 1-4 \beta\rangle$. The expression we should minimize according to LLM (p. 296) is thus just a function of $\beta$ and is equal to the following:

$$
-0.25 \cdot \ln (3 \beta)-0.25 \cdot \ln \beta-0.5 \cdot \ln (1-4 \beta)
$$

8. Observe that this is equivalent, since $R_{1}$ and $R_{2}$ are disjoint, to the evidence entailing (only) a specific new conditional credence. In the original example Judy learns that $b^{\prime}\left(R_{1} \mid R_{1} \vee\right.$ $\left.R_{2}\right)=3 / 4$, which (if $b^{\prime}\left(R_{1}\right)$ and $b^{\prime}\left(R_{2}\right)$ are not equal to o) is equivalent to $b^{\prime}\left(R_{1}\right)=3 \cdot b^{\prime}\left(R_{2}\right)$.

9. I omit the arguments for this and the previous observation. They are similar to the proofs of Facts 1 and 2 below given in the Appendix.

10. I am unable to give a general account of this cardinality dependence. For the purposes of the current paper it is enough to notice that the dependence exists. 
the derivative of which is

$$
-\frac{0.25}{\beta}-\frac{0.25}{\beta}+\frac{2}{1-4 \beta}
$$

which equals 0 exactly when

$$
\beta=0.125
$$

where (since the second derivatives are positive) the expression (I) reaches its minimum. And so the belief function minimizing expected inaccuracy as given by the logarithmic measure is $\langle 0.375,0.125,0.5\rangle$, which means that $b^{\prime}(B)=b(B)=$ 1/2. A simple Lagrangian Multiplier argument extends this to cases of arbitrary finite cardinality of all propositions involved.

This is not a novel finding, but an easy (I think) way of showing a result by Douven and Romeijn (2011), who found that the MIRE method does give the "intuitive" solution to the Judy Benjamin problem. Since we have already observed that LLM is equivalent to MIRE, the "good" behavior of LLM shown above should not be surprising.

So far, then, we have seen that the LP framework's answer to the Judy Benjamin problem is "unintuitive" in the same sense in which MRE's answer is: the degree of belief in a proposition which is not explicitly talked about is supposed to increase. In addition, the answers mandated by the LP framework are cardinality dependent, as opposed to those given by MRE. MIRE offers both the intuitive and cardinality independent solution to the problem. It also has the pleasing feature of giving a graspable reduction of the Judy Benjamin problem to one solvable by Jeffrey Conditionalization; "graspable" in the sense that it can be given only by referring to old credences and new constraints, as we will see shortly. In contrast, any application of MRE to the Judy Benjamin problem with a particular $m$ arrives at a belief function which is also obtainable using Jeffrey Conditionalization, but no "graspable" reduction seems to exist.

\section{The rule which MIRE endorses for the Judy Benjamin problem:}

Evidence: entails (only), for some disjoint propositions $A$ and $B$ and a nonzero positive real number $m$, that $b^{\prime}(A)=m \cdot b^{\prime}(B)$.

Response: Consider this as a case in which the evidence entails (only) new credences in all elements of the partition $\{A, B, \neg(A \vee B)\}$ :

- $b^{\prime}(\neg(A \vee B))=b(\neg(A \vee B))$;

- $b^{\prime}(B)=\frac{b(A \vee B)}{m+1}$;

- $b^{\prime}(A)=1-b^{\prime}(\neg(A \vee B))-b^{\prime}(B)$

and use the JC rule. 
If we generalize the evidential situation so that it involves an arbitrary number of finite disjoint propositions, we will see that MIRE endorses a rule called "Adams Conditionalization", which is a special case of JC (see Bradley 2005).

In what follows, I will refer to the evidence which features in the Judy Benjamin problem, namely, evidence which entails (only) a new conditional credence, or equivalently a proportion of credences in two disjoint propositions, by the name "Judy Benjamin evidence".

\section{The Simultaneous Update Problem}

We will now turn to the second belief update problem to be discussed. It boils down to the following: what is one's rational posterior belief function if one's evidence entails (only) one's new credences in overlapping propositions? (I will call such evidence "overlapping evidence".) That is, suppose you know of your new belief function $b^{\prime}$ that $b^{\prime}(A)=p$ and $b^{\prime}(B)=q$ for some $p$ and $q$, but as a matter of fact $A \cap B \neq \varnothing$. What is the precise shape your $b^{\prime}$ should take?

Notice that the situation does not allow us to use JC (or AJC) right away: we are not given new credences in all elements of some partition. What we do receive are new credences in all elements of two partitions: $\{A, \neg A\}$ and $\{B, \neg B\}$. One direction in which we could proceed would be to argue for some transformation of the data entailed by the evidence so that new credences in all elements of a single partition would be obtained; seemingly the most natural partition to be used here consists of the four logical combinations of $A$ and $B$. If this was successful, we could then use an appropriate update rule-e.g., JC or AJC - compatible with our chosen belief update method. I will argue below that in the case of the LP framework such an approach is only partially successful, while the goal is obtained with no reservations if we use MIRE.

Why is the problem called one of "simultaneous update"? This is because it relates to a well known feature of Jeffrey Conditionalization (shared by AJC): its noncommutativity. JC is sensitive to the order of applying it to numerical constraints following from the evidence. That is, applying JC twice, using two sets of new credences in elements of some two partitions, will typically lead to a different belief function depending on which set is used first. Some people consider this to be a problem, since the new credences are taken to correspond to the evidence received, and the order of evidence should - they say-not matter for the eventual belief function. In my opinion this worry is insubstantial because changing the order of using the numerical constraints usually has to change the evidence from which these constraints are supposed to follow. (Think about the different evidence needed to change your degree of belief that the coin you are repeatedly tossing is fair to 0.9 depending on whether your initial credence in that is 0.01 or 0.8 .) This is not an original view-Lange (2000) and Osherson 
(2002) present arguments to the effect that changing the order of application of the purely numerical constraints results in describing two different evidential situations. ${ }^{11}$ Still, there have been attempts at combining the data about two partitions into one, thus reducing two updates to be performed consecutively into a single "simultaneous" one. An example of this is the recent paper by Park (2013), where the author analyses in detail an earlier suggestion from Williams (1980) regarding the application of MRE to that task. Subsection 3.2 of the current paper can be seen as complementing the results of Park (2013), using MIRE instead.

\subsection{How QUM Approaches the Problem}

We will first consider the simplest non-trivial case, which involves four possible worlds, and two two-world propositions sharing one world. Notice that in this case the task reduces to figuring out $b^{\prime}(A \cap B)$. It turns out that the LP framework, with its choice of QUM as its belief update method, offers a simple solution to the simultaneous update problem in this case (see the Appendix for the proof, p. 314, but remember the note on notation just before the argument):

Fact 1 Let $W=\left\{w_{1}, w_{2}, w_{3}, w_{4}\right\}$ be the set of epistemically possible worlds for some agent. Let $A$ be the proposition $\left\{w_{1}, w_{2}\right\}$; let $B$ be $\left\{w_{2}, w_{3}\right\}$. Suppose that the agent's evidential constraints are that her posterior belief function $b^{\prime}$ satisfies the following: $b^{\prime}(A)=p$ and $b^{\prime}(B)=q$, for some $p$ and $q$. Let us label the number $b(A \cap B)+$ $\frac{p-b(A)+q-b(B)}{2}$ as $K$. The belief update function which the agent should adopt according to QUM is fully determined by the two constraints and the following condition:

$$
b^{\prime}(A \cap B)= \begin{cases}0 & \text { if } K<0 \\ \min \{p, q\} & \text { if } \min \{p, q\}<K \\ K & \text { otherwise. }\end{cases}
$$

That is, the change in your credence in $A \cap B$ is the average of the changes in your credences in $A$ and in $B$ (whenever it makes mathematical sense, i.e., you don't go above 1 or below 0 ). I would say that the solution is at least not immediately absurd. I will now illustrate it by an example using a modified story from Osherson (2002).

Suppose that listening to the radio I hear a forecast for rain but I'm not sure whether it comes from the chief meteorologist or from his unreliable deputy. After the broadcast concludes I have the following belief function $b_{1}$, where $R$ is "It rains today" and $C$ is "The chief was speaking":

11. This does not put the issue fully to rest. See Weisberg (2009) on "commutativity on experiences". 


\begin{tabular}{c|cccc} 
& $\neg R C$ & $R C$ & $R \neg C$ & $\neg R \neg C$ \\
\hline$b_{1}$ & .2 & .4 & .1 & .3
\end{tabular}

Now, a glance at the sky raises my credence in $R$ to .7. Suppose also that the forecast is rebroadcasted and even though I strain my ears, I conclude I should not change my credence in $C$. The following is my new credence as dictated by Fact 1 .

\begin{tabular}{c|cccc} 
& $\neg R C$ & $R C$ & $R \neg C$ & $\neg R \neg C$ \\
\hline$b_{1}^{\prime}$ & .1 & .5 & .2 & .2
\end{tabular}

This is because my credence in $R$ increases by .2, and my credence in $C$ stays the way it was. The average of these changes is .1, which according to Fact 1 is the increase in my credence in $R C$. In this simple case all other credences are directly calculable.

Even though the solution of the problem offered by QUM in the simplest nontrivial case may look reasonable, I would like to raise some problems regarding it:

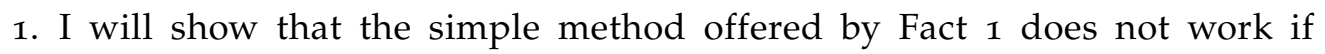
the cardinality of the events is different; that is, in the LP framework the information entailed by the agent's evidence is not directly translatable into information about a partition (Section 3.1.1);

2. I will also argue that already in the 4-world case the updating rule dictated by the LP framework leads to unfortunate updating behaviour (Section 3.1.2.

\subsubsection{Cardinality Dependence Again}

Given Fact I, at this point a prima facie reasonable way to proceed, inside the LP framework, would be the following:

- given any similar situation with an arbitrary finite $W$, calculate $b^{\prime}(A \cap B)$ using the above formula;

- this, together with the two constraints, would presumably give us the posterior credences in all propositions from the set $\{A \cap B, A \cap \neg B, \neg A \cap B, \neg A \cap$ $\neg B$, which is a partition of $W$ : in this way we would obtain constraints such as would follow from Jeffrey evidence;

- having that, we should now (it would seem) use AJC to derive the full shape of $b^{\prime}$ (since we know this is the rule QUM leads to in response to Jeffrey evidence). 
It turns out, however, that the LP framework itself would judge this procedure as wrong. It turns out the answer depends on the cardinality of the propositions. We already knew that cardinality is a factor playing an important role for AJC; we will now see that in situations involving new credences in two overlapping propositions cardinality is relevant also for the input for AJC; depending on the cardinality of the propositions involved, the new credences in the four logical combinations of the two propositions will be different. I think that it is an unfortunate consequence which we should try to avoid. I will now illustrate it by the following Fact and a modification of the previously used weather forecasting example. The relevant feature of the LP framework is the following (for the proof and some discussion of the mathematical details see again the Appendix, p. 314 . but remember the note on notation on $p \cdot 313$ :

Fact 2 Let $W=\left\{w_{0}, w_{1}, w_{2}, w_{3}, w_{4}\right\}$ be the set of epistemically possible worlds for some agent. Let $A$ be the proposition $\left\{w_{0}, w_{1}, w_{2}\right\}$; let $B$ be $\left\{w_{2}, w_{3}\right\}$. Suppose that the agent's evidential constraints are that her posterior belief function $b^{\prime}$ satisfies the following: $b^{\prime}(A)=p=b(A)$ and $b^{\prime}(B)=q$, for some $p$ and $q$. If the belief update function which the agent adopts is the one fully determined by the two constraints and the following condition

$$
\begin{aligned}
b^{\prime}(A \cap B) & =b(A \cap B)+4 / 7(q-b(B)), \\
b^{\prime}\left(\left\{w_{0}\right\}\right) & =b\left(\left\{w_{0}\right\}\right)-2 / 7(q-b(B)), \\
b^{\prime}\left(\left\{w_{1}\right\}\right) & =b\left(\left\{w_{1}\right\}\right)-2 / 7(q-b(B)),
\end{aligned}
$$

then it is the one which QUM dictates the agent should adopt in this case.

I do not want to suggest that this is in any way a profound discovery: it is a result of a simple minimization argument which will allow me to produce examples which point, in my opinion, to some deficiencies of QUM.

Consider, then, a modification of the previous story about the chief meteorologist and the weather. The goal is now to split one possible world into two worlds; to transform the previous four-world case to an example where the proposition $\neg R C$ contains two worlds and so the whole space contains five worlds. Suppose, then, that I owe the chief meteorologist money and I don't want to meet him. I know that whenever it doesn't rain, he always walks home through a park he never visits otherwise and in which I walk my dog (which I do regardless of the weather); not wanting to disturb my dog's routine, I will also go to the park today. (If it rains, the chief meteorologist takes a bus home.) The chief meteorologist can traverse the park via one of two paths, call them " 1 " and " 2 ". Let $B$ be the proposition "the chief meteorologist will walk via path 1 today". My four epistemic possible worlds

$$
\neg R C \quad R C \quad R \neg C \quad \neg R \neg C
$$


become

$$
\neg R C B \quad \neg R C \neg B \quad R C \neg B \quad R \neg C \neg B \quad \neg R \neg C \neg B
$$

and my initial credence becomes, say,

$$
\begin{array}{c|ccccc} 
& \neg R C B & \neg R C \neg B & R C \neg B & R \neg C \neg B & \neg R \neg C \neg B \\
\hline b_{2} & .1 & .1 & .4 & .1 & .3
\end{array}
$$

(it is not important that my credences in $\neg R C B$ and $\neg R C \neg B$ are equal: they just need to sum up to .2). Notice that the $\neg R C$ world has effectively split in two; think of the two stories as describing two agents with a similar but different space of epistemically possible worlds.

Consider now the same evidential situation and suppose (which I think is intuitive enough ${ }^{12}$ ) that it gives rise to the same numerical constraints: a glance at the sky raises my credence in $R$ to .7; the forecast is rebroadcasted and even though I strain my ears I conclude I should not change my credence in $C$. The following is, by Fact 2 . my new credence, as mandated by QUM:

$$
\begin{array}{c|ccccc} 
& \neg R C B & \neg R C \neg B & R C \neg B & R \neg C \neg B & \neg R \neg C \neg B \\
\hline b_{2}^{\prime} & .043 & .043 & .514 & .186 & .214
\end{array}
$$

Now recall the initial and updated credences for the first agent:

\begin{tabular}{c|cccc} 
& $\neg R C$ & $R C$ & $R \neg C$ & $\neg R \neg C$ \\
\hline$b_{1}$ & .2 & .4 & .1 & .3 \\
$b_{1}^{\prime}$ & .1 & .5 & .2 & .2
\end{tabular}

Comparing $b_{1}^{\prime}$ and $b_{2}^{\prime}$ we notice, then, that the two agents:

- started with the same credences in $R, C$, and $R C ;{ }^{13}$

- faced the same evidence implying (only) that they should increase their credence in $R$ by the same amount and not to change their credence in $C$;

- ended with a different credence in $R C$;

- and it seems the only difference was whether the park was considered or not.

12. Although I realise that for some it would need an additional argument regarding context-(in)sensitivity and related issues. I hope to tackle these topics in a future paper.

13. This is not literally true, e.g. since $C$ is for one agent a doubleton, and for the other a three-element set. To be technically correct I would have to write something like "the agents started with the same credences in the propositions (sets of worlds) they associate with the sentence 'the chief was speaking'"; I decided to avoid the verbiage. 
In my opinion this updating behavior is unintuitive: the role of the park is unclear at best. It would seem to me that we should avoid this kind of cardinality dependence if we can; in the next section I will show that the MIRE methods succeeds in this. I also do not see a way of invoking context-dependence, which for some is a natural way of replying to similar cardinality-dependence-related issues troubling Leitgeb's (2014) stability theory.

There are also cases in which not only the credence in a suitably chosen proposition ends up being different, but the two agents end up with different probability rankings regarding two propositions which they initially believe to the same degree (and so rank in the same way): that is, cases in which for some two propositions $\phi$ and $\psi$

$$
b_{1}(\phi)=b_{2}(\phi) \text { and } b_{1}(\psi)=b_{2}(\psi)
$$

but

$$
b_{2}^{\prime}(\phi)<b_{2}^{\prime}(\psi) \text { while } b_{1}^{\prime}(\phi)>b_{1}^{\prime}(\psi) .
$$

As an example, consider two agents with sets of epistemically possible worlds and propositions as discussed above, and suppose the initial belief functions of the agents look like this:

\begin{tabular}{c|c|ccccc} 
& & $\neg R C$ & $R C$ & $R \neg C$ & $\neg R \neg C$ \\
\cline { 2 - 6 } & & .21 & .3 & .3 & .19 & \\
& & $\neg R C B$ & $\neg R C \neg B$ & $R C \neg B$ & $R \neg C \neg B$ & $\neg R \neg C \neg B$ \\
\hline$b_{2}$ & .105 & .105 & .3 & .3 & .19
\end{tabular}

Notice that $b_{1}(\neg R C)=.21=b_{2}(\neg R C)$ and $b_{1}(\neg R \neg C)=.19=b_{2}(\neg R \neg C)$. So both agents initially rank $\neg R C$ as more probable than $\neg R \neg C$. Suppose now that the empirical constraints dictate that the credence in $C$ is to stay the same, while the credence in $R$ is to become .9. The following tables show the posterior belief functions of the two agents as calculated using Facts 1 and 2

$$
\begin{aligned}
& \begin{array}{c|cccc} 
& \neg R C & R C & R \neg C & \neg R \neg C \\
\hline b_{1}^{\prime} & .06 & .45 & .45 & .04
\end{array} \\
& \begin{array}{c|ccccc} 
& \neg R C B & \neg R C \neg B & R C \neg B & R \neg C \neg B & \neg R \neg C \neg B \\
\hline b_{2}^{\prime} & .019 & .019 & .472 & .428 & .062
\end{array}
\end{aligned}
$$

Notice that $b_{1}^{\prime}(\neg R C)=.06>.04=b_{1}^{\prime}(\neg R \neg C)$, while $b_{2}^{\prime}(\neg R C)=.038<.062=$ $b_{2}^{\prime}(\neg R \neg C)$. So despite the fact that the agents received the same empirical constraints regarding their new credences in $R$ and $C$, one of them reversed her ranking of two logical combinations of those propositions. 


\subsubsection{QUM: The Four-World Cases}

Cardinality issues aside, I will now argue that Fact 1 alone already has some unintuitive consequences pointing at deficiencies of the LP framework. Consider a different modification of our original story involving an agent with the initial belief function $b_{1}$ ( $\mathrm{p}$. 305). Suppose two pieces of evidence are given:

- a glance at the sky lowers the credence in $R$ to .4 (by .1);

- a rebroadcasting of the forecast increases the credence in $C$ to .7 (by .1).

The following is the new credence as dictated by Fact $\mathrm{T}$;

\begin{tabular}{c|cccc} 
& $\neg R C$ & $R C$ & $R \neg C$ & $\neg R \neg C$ \\
\hline$b_{1}^{\prime \prime}$ & .3 & .4 & .0 & .3
\end{tabular}.

Because the average of the two changes is 0 , the credence in the conjunction has to stay the same. Notice that the agent has ended up with credence 0 - that is, the lowest possible for a probabilist-in $R \neg C$, for unknown reasons. That by itself is unintuitive, but situations in which similar phenomena arise were already described by Levinstein (2012). I want to add to this an interesting feature of the updating behavior which follows from Fact 1 . What happened in the last example is that the agent updated her credences in $R$ and $C$ as if these two were perfectly anticorrelated; that is, she interpreted an increase in the credence in $C$ as an increase in the credence in $\neg R C$, and she interpreted a decrease in the credence in $R$ as a decrease in the credence in $R \neg C$. She acted as if she was either oblivious of the fact that she holds a positive credence in $R C$, or she thought the influence on her credence in $R C$ of the two pieces of evidence exactly cancels out, which should be supported by some additional argument.

In general, we can notice that Fact 1 dictates the following update behavior:

- if increase in the credence in $R$ equals the increase in the credence in $C$ : update as if they are perfectly correlated (increase the credence in $R C$ by the same amount);

- if increase in the credence in $C$ equals the negative of the increase in the credence in $R$ : update as if they are perfectly anticorrelated (leave the credence in $R C$ as it was, increase the credence in $\neg R C$ and decrease the credence in $R \neg C$ by the same amount),

whenever it makes mathematical sense (no credences end up being negative, etc.)

Even if I think that the main disadvantage of QUM presented in this section is the cardinality dependence (Subsection 3.1.1), the updating behavior just outlined may also be worrying: I would say it is not obvious and definitely requires 
some supporting argument. I will also note that this behavior extends to some extent beyond the four-world case, but I only calculated some specific five-world examples.

We will now see that using the MIRE method allows us to avoid the cardinality dependence described above.

\subsection{The MIRE Solution to the Problem}

It turns out that the MIRE method (p. 296), or equivalently the local-logarithmic method, offers a solution to the simultaneous update problem which is independent of cardinality of the propositions involved; that is, the problem is reduced to figuring out the credences in the four logical combinations of the propositions, at which point Jeffrey Conditionalization is invoked (for the proof see again the Appendix, p. 316, and again remember the note on notation on p. 313

Fact 3 Let $A$ and $B$ be two propositions such that $A \cap B \neq \varnothing$ and let $b$ be the agent's prior belief function. Suppose that the agent's evidential constraints entail (only) her new credences $b^{\prime}(A)$ and $b^{\prime}(B)$. To arrive at the belief function $b^{\prime}$ the agent should adopt according to MIRE we should first calculate the agent's new credences in the logical combinations of $A$ and $B$ so that the following is true

$$
\frac{b(A \cap \neg B)}{b^{\prime}(A \cap \neg B)}+\frac{b(\neg A \cap B)}{b^{\prime}(\neg A \cap B)}=\frac{b(A \cap B)}{b^{\prime}(A \cap B)}+\frac{b(\neg A \cap \neg B)}{b^{\prime}(\neg A \cap \neg B)}
$$

and then use Jeffrey Conditionalization.

If we write the new credences in $A$ and $B$ as $p$ and $q$, we can rewrite the condition in Fact 3 as

$$
\frac{b(A \cap \neg B)}{p-b^{\prime}(A \cap B)}+\frac{b(\neg A \cap B)}{q-b^{\prime}(A \cap B)}=\frac{b(A \cap B)}{b^{\prime}(A \cap B)}+\frac{b(\neg A \cap \neg B)}{b^{\prime}(A \cap B)+1-p-q}
$$

at which point it is more immediately seen that what we have is just an equation with a single variable. Moreover, the solution-that is, the value of $b^{\prime}(A \cap B)$ is always available for the agent (see the proof in the Appendix). I would say that an intuitive advantage of this solution-apart from what I take to be its modest mathematical elegance, though this of course is a matter of taste-is that the new credences in the four logical combinations of $A$ and $B$ are independent of the cardinalities of the propositions, and so no such unfortunate updating behavior as the one described in Section 3.1.1 can arise. This for me forms another argument in favor of using the MIRE update method instead of the quadratic one. Just to reiterate, in my opinion MIRE also has the edge over MRE because it delivers the "intuitive" solution to the Judy Benjamin problem.

The difference between the MIRE method and QUM is already visible in our original, four-world updating case: as the reader may verify, the eventual 
belief function $b_{1_{\text {MIRE }}^{\prime}}^{\prime}$ dictated by the MIRE update method is as approximately displayed in the following table:

\begin{tabular}{l|cccc} 
& $\neg R C$ & $R C$ & $R \neg C$ & $\neg R \neg C$ \\
\hline$b_{1}$ & .2 & .4 & .1 & .3 \\
$b_{1}^{\prime}$ & .1 & .5 & .2 & .2 \\
$b_{1_{\text {MIRE }}^{\prime}}^{\prime}$ & 0.107 & 0.493 & 0.207 & 0.193
\end{tabular}

The new credences allotted by MIRE to the four propositions will be as above regardless of how many (but finitely many) worlds belong to each of them. The way of updating mandated by MIRE will also not exhibit the worrying features described on p. 309 ("updating as if the events were perfectly anticorrelated", etc.)

To connect this development with the results in Park (2013) I will now present a Fact containing a direct formula for the posterior belief function mandated by MIRE in response to the simultaneous update problem concerning some two propositions $A$ and $B$. It will be convenient to use characteristic functions. Define, then, for any $w_{i} \in W$ and $E \in\{A, B\}$,

$$
\chi_{E, i}=\left\{\begin{array}{l}
1 \text { if } w_{i} \in E \\
0 \text { otherwise }
\end{array}\right.
$$

Fact 4 Let $A$ and $B$ be two propositions such that $A \cap B \neq \varnothing$ and let $b$ be the agent's prior belief function. Suppose that the agent's evidential constraints entail (only) her new credences $b^{\prime}(A)=p$ and $b^{\prime}(B)=q$. The belief function $b^{\prime}$ the agent should adopt according to MIRE is defined as follows:

For any $i \in\{1, \ldots, n\}, b^{\prime}\left(\left\{w_{i}\right\}\right)=\frac{b\left(\left\{w_{i}\right\}\right)}{-\lambda_{0}-\lambda_{1} \chi_{A, i}-\lambda_{2} \chi_{B, i}}$ with parameters $\lambda_{0}, \lambda_{1}$ and $\lambda_{2}$ determined by the following three equations:

$$
\begin{gathered}
\sum_{i=1}^{n} \frac{b\left(\left\{w_{i}\right\}\right)}{-\lambda_{0}-\lambda_{1} \chi_{A, i}-\lambda_{2} \chi_{B, i}}=1 \\
\sum_{i=1}^{n} \chi_{A, i} \frac{b\left(\left\{w_{i}\right\}\right)}{-\lambda_{0}-\lambda_{1} \chi_{A, i}-\lambda_{2} \chi_{B, i}}=p \\
\sum_{i=1}^{n} \chi_{B, i} \frac{b\left(\left\{w_{i}\right\}\right)}{-\lambda_{0}-\lambda_{1} \chi_{A, i}-\lambda_{2} \chi_{B, i}}=q .
\end{gathered}
$$

For a proof see the Appendix (p. 318). Unfortunately neither Fact 3 nor 4 is easily generalizable either with respect to the number of partitions or the number of elements of the partitions.

One benefit of formulating the results in the above form is that it can immediately be seen that updating in the way mandated by MIRE is equivalent to updating 
using Jeffrey Conditionalization on the more "fine-grained" partition $\{A, \neg A\} \times$ $\{B, \neg B\}$. One way to see this is to notice that, if MIRE is used, then for any cell $c$ of the more fine-grained partition there is a number $r_{c}$ such that for any world $w_{i}$ inside that cell $b^{\prime}\left(\left\{w_{i}\right\}\right)=b\left(\left\{\omega_{i}\right\}\right) \cdot r_{c}$, which is characteristic of Jeffrey Conditionalization. After consulting the formulas in Park 2013: 3517) we see that the same is true for updating using MRE.

For those who would like to defend JC against the criticism of noncommutativity (p. 303) by presenting a way of reducing the two consecutive updates to one there are then at least two options, one using MRE (see Park 2013), the other using MIRE (presented here). ${ }^{14}$ Which of them is better has to depend on the specifics of the given situation and on the interpretation of the relative entropy expressions the two methods employ. I leave investigating these issues for a future paper.

In this section I tried first to show what QUM says about the simultaneous update problem in certain types of cases, to argue next that this points to some deficiencies of the LP framework. I then showed that MIRE provides a cardinalityindependent solution, which also allows adherents of Jeffrey Conditionalization to respond to some versions of the criticism regarding the rule's noncommutativity.

\section{Conclusions}

The impulse for the paper was to respond to two open problems regarding belief update in the LP framework, which employs the quadratic update method. I have given partial answers in sections 2 and 3 . I tried to argue that the answers exhibit some troubling features of QUM, most importantly a sort of cardinalitydependence which has not been, as far as I know, discussed in the literature (Section 3.1.1) and not giving the "intuitive" (p. 300) solution to the Judy Benjamin problem (Section 2). Along the way I tried to show that the method employing inverse relative entropy fares better in that regard.

The following table, which sums up the results of this paper, displays the status ("acceptable" / "not acceptable") of the three belief update methods's responses to the considered types of evidence from the perspective of someone who prefers the "intuitive" solution to the Judy Benjamin problem and cardinalityindependent solutions to belief update problems in general:

14. Unfortunately, as mentioned before, in this paper I only show the way MIRE deals with the issue if two partitions with two elements are involved. Park describes also the case of three two-element partitions. 


\begin{tabular}{||c|c|c|c||}
\hline \hline & $\begin{array}{c}\text { Jeffrey } \\
\text { evidence }\end{array}$ & $\begin{array}{c}\text { overlapping } \\
\text { evidence }\end{array}$ & $\begin{array}{c}\text { Judy Benjamin } \\
\text { evidence }\end{array}$ \\
\hline \hline QUM & $\mathrm{X}$ & $\mathrm{X}$ & $\mathrm{X}$ \\
\hline MRE & $\checkmark$ & $\checkmark$ & $\mathrm{X}$ \\
\hline MIRE & $\checkmark$ & $\checkmark$ & $\checkmark$ \\
\hline \hline
\end{tabular}

I do not want to suggest that MIRE trumps all other belief update methods, full stop. My goal was to argue that there are situations in which MIRE works at least as well as MRE, while QUM leads to unintuitive results. Of course, intuitions vary. Those, who-like van Fraassen-were dissatisfied with MRE's approach to the Judy Benjamin problem, and those who prefer cardinality independent solutions to belief update problems, should, I think, be relatively happy with what MIRE offers. A detailed investigation of the differences between the methods of MRE and MIRE with analysis of their applicability in various situations will have to wait for a future paper-this one is already too long.

\section{Appendix}

A note on notation: In all facts below we will be speaking about propositions $A$ and $B$. There will be a finite number of possible worlds $w_{1}, \ldots, w_{n}$ (and in one case also $w_{0}$ ). Prior credences in those will be denoted by $v_{1}, \ldots, v_{n}$ (and in one case also $v_{0}$ ). For clarity (I hope) in the proofs below we will denote posterior credences in singletons of elements of $A \cap \neg B$, in ascending order of the indices, by $a_{0}, \ldots, a_{m}$; in the trivial one-element case we will just use $a$. (In fact, below we will only explicitly write out one proof using just $a_{0}$ and $a_{1}$ ). The posterior credence in $B \cap \neg A$ will be denoted by $b$; that in $A \cap B$ by $k$ (for kommon), while that in $\neg A \cap \neg B$ by $z$. We will not use subscripts for $b, k$, and $z$, since in the proofs below the cases in which the three corresponding logical combinations of $A$ and $B$ are involved as consisting of more than one possible world will be suppressed; when written out in full, subscripts would be needed just like in the case of $a$.

Proof of Fact 1

Fact 1 Let $W=\left\{w_{1}, w_{2}, w_{3}, w_{4}\right\}$ be the set of epistemically possible worlds for some agent. Let $A$ be the proposition $\left\{w_{1}, w_{2}\right\}$; let $B$ be $\left\{w_{2}, w_{3}\right\}$. Suppose that the agent's evidential constraints are that her posterior belief function $b^{\prime}$ satisfies the following: $b^{\prime}(A)=p$ and $b^{\prime}(B)=q$, for some $p$ and $q$. Let us label the number $b(A \cap B)+$ $\frac{p-b(A)+q-b(B)}{2}$ as $K$. The belief update function which the agent should adopt according 
to QUM is fully determined by the two constraints and the following condition:

$$
b^{\prime}(A \cap B)= \begin{cases}0 & \text { if } K<0 \\ \min \{p, q\} & \text { if } \min \{p, q\}<K \\ K & \text { otherwise. }\end{cases}
$$

Proof: The expected inaccuracy of a belief function $b^{\prime}$ as given by the quadratic measure is as follows:

$$
\sum_{i=1}^{4} v_{i}\left\|w_{i}-b^{\prime}\right\|^{2}
$$

which reaches its minimum for the same argument as

$$
\underbrace{\left(v_{1}+\cdots+v_{4}\right)}_{1}\left(a^{2}+\cdots+z^{2}\right)-2 v_{1} a-2 v_{2} k-2 v_{3} b-2 v_{4} z .
$$

Using the equalities $a=p-k, b=q-k, z=1+k-p-q$ we can write this expression as a function of $k$ only (since $p$ and $q$ are given). After expansion and some tedious but straightforward calculation (using also the equality $v_{4}=$ $\left.1-\left(v_{1}+v_{2}+v_{3}\right)\right)$ we see that it reaches its minimum for the same argument as

$$
k^{2}+\left(v_{1}+v_{3}-p-q\right) k
$$

The derivative of (2) is an increasing linear function reaching 0 at

$$
k=\frac{p-v_{1}+q-v_{3}}{2}
$$

and so (2) is minimized for this value of $k$. We leave it to the reader to show that $k=K$ where $K$ is as specified in Fact 1 .

Now, the value of $k$ as given by (2) might turn out to be negative or higher than $p$ or $q$, and thus be unavailable to the agent. Since, as noted, the derivative of (2) is an increasing linear function, in these cases to minimize expected inaccuracy the agent should set $k$ to 0 or to the lower of the values $p$ and $q$, accordingly. Q.E.D.

Proof of Fact 2

Compared to the previous proof, this one adds one possible world to the proposition $A \cap \neg B$; we will index it as well as the prior credence in its singleton by 0 .

Fact 2 Let $W=\left\{w_{0}, w_{1}, w_{2}, w_{3}, w_{4}\right\}$ be the set of epistemically possible worlds for some agent. Let $A$ be the proposition $\left\{w_{0}, w_{1}, w_{2}\right\}$; let $B$ be $\left\{w_{2}, w_{3}\right\}$. Suppose that the agent's evidential constraints are that her posterior belief function $b^{\prime}$ satisfies the following: $b^{\prime}(A)=p=b(A)$ and $b^{\prime}(B)=q$, for some $p$ and $q$. If the belief update 
function which the agent adopts is the one fully determined by the two constraints and the following condition

$$
\begin{aligned}
b^{\prime}(A \cap B) & =b(A \cap B)+4 / 7(q-b(B)), \\
b^{\prime}\left(\left\{w_{0}\right\}\right) & =b\left(\left\{w_{0}\right\}\right)-2 / 7(q-b(B)), \\
b^{\prime}\left(\left\{w_{1}\right\}\right) & =b\left(\left\{w_{1}\right\}\right)-2 / 7(q-b(B)),
\end{aligned}
$$

then it is the one which QUM dictates the agent should adopt in this case.

Notice that the main claim of Fact 2 is (only) an implication: this is because here it is less convenient to deal with the cases in which it would seem from initial calculations that one's credence in a proposition should be negative than in the four-world case; we are not concerned with such examples. This is similar to the problems Leitgeb and Pettigrew have to deal with in their proof that QUM dictates that AJC is the method of updating in response to Jeffrey evidence. We are interested only in the "favorable" cases, because we want to use them to illustrate some deficiencies of the QUM method.

Notice also that this is a case of AJC updating: the change in credence in each element of $A \cap \neg B$ is obtained by decreasing the prior credence by the same number.

Proof: The change from the proof of Fact 1 is that instead of a single $a$ we now have to consider $a_{0}$ and $a_{1}$. The expected inaccuracy of the considered belief function reaches its minimum for the same argument as the following expression:

$$
\underbrace{\left(v_{0}+\cdots+v_{4}\right)}_{1}\left(a_{0}^{2}+\cdots+z^{2}\right)-2 v_{0} a_{0}-2 v_{1} a_{1}-2 v_{2} k-2 v_{3} b-2 v_{4} z .
$$

Using the equalities $a_{0}=p-k-a_{1}, b=q-k, z=1+k-p-q$ we can write this expression as a function of $k$ and $a_{1}$ only. After expansion and some more tedious but still straightforward calculation we see that it reaches its minimum for the same arguments as

$$
2 k^{2}+\left(a_{1}-2 p-2 q+2 v_{0}+v_{1}+2 v_{3}\right) k+a_{1}^{2}+\left(v_{0}-v_{1}-p\right) a_{1}
$$

which after using the equality $p=v_{0}+v_{1}+v_{2}$ (true since we assumed the the credence in $A$ does not change) transforms into

$$
2 k^{2}+\left(a_{1}-2 q-v_{1}-2 v_{2}+2 v_{3}\right) k+a_{1}^{2}+\left(-2 v_{1}-v_{2}\right) a_{1} .
$$

(3) is a function of two variables, $k$ and $a_{1}$. Its partial derivative w.r.t. $a_{1}$ reaches 0 for $a_{1}=\frac{2 v_{1}+v_{2}-k}{2}$; when we plug this into the partial derivative w.r.t. $k$ and assume it equals 0 (since we are looking for a minimum) we arrive at

$$
7 k=4 q+3 v_{2}-4 v_{3}
$$


which is equivalent to

$$
k=v_{2}+4 / 7\left(q-v_{2}-v_{3}\right),
$$

that is, to

$$
b^{\prime}(A \cap B)=b(A \cap B)+4 / 7(q-b(B))
$$

as required. The other two conditions of Fact 2 , on $b^{\prime}\left(\left\{w_{0}\right\}\right)$ and $b^{\prime}\left(\left\{w_{1}\right\}\right)$, follow now by straightforward calculation (remember that the credence in $A$ does not change). Q.E.D.

The next proof is a bit lengthy, but does not use the method of Lagrange multipliers. Fact 3 contains a more approachable description of the belief update function which is fully specified in Fact 4. For that Fact I give below a concise proof using Lagrange multipliers, so if the reader is familiar with the method, it might be better to go there (p. 318).

Proof of Fact 3

Fact 3 Let $A$ and $B$ be two propositions such that $A \cap B \neq \varnothing$ and let $b$ be the agent's prior belief function. Suppose that the agent's evidential constraints entail (only) her new credences $b^{\prime}(A)$ and $b^{\prime}(B)$. To arrive at the belief function $b^{\prime}$ the agent should adopt according to MIRE we should first calculate the agent's new credences in the logical combinations of $A$ and $B$ so that the following is true

$$
\frac{b(A \cap \neg B)}{b^{\prime}(A \cap \neg B)}+\frac{b(\neg A \cap B)}{b^{\prime}(\neg A \cap B)}=\frac{b(A \cap B)}{b^{\prime}(A \cap B)}+\frac{b(\neg A \cap \neg B)}{b^{\prime}(\neg A \cap \neg B)}
$$

and then use Jeffrey Conditionalization.

Proof: First, in what follows we will use the following simple observation.

Observation 1 Suppose that, for some natural number $j, x_{0}, \ldots, x_{j}$ and $y_{0}, \ldots, y_{j}$ are strictly positive real numbers. If $\forall i \in\{1, \ldots, j\} \frac{x_{0}}{y_{0}}=\frac{x_{i}}{y_{i}}$, then $\frac{x_{0}}{y_{0}}=\frac{\sum_{i=0}^{j} x_{i}}{\sum_{i=0}^{j} y_{i}}$.

Second, remember that it can be shown by a limit argument that according to MIRE the agent should use Jeffrey Conditionalization once the new credences in the four logical combinations of $A$ and $B$ are determined (see Pettigrew, 2016, and Diaconis and Zabell, 1982).

We need to show that the relationship (4) holds independently of the number of atoms in the probability space. We will first show that it holds in the simplest, four-world case, and then show that adding a finite number of worlds to each of the logical combinations of the two propositions does not break the relationship. Since the MIRE method is equivalent to the local-logarithmic one (p. 297), we will use the latter for calculations.

The four-world case. The local-logarithmic method tells us we need to minimize the following expression containing the single variable $k$ :

$$
-v_{1} \cdot \ln (p-k)-v_{2} \cdot \ln k-v_{3} \cdot \ln (q-k)-v_{4} \cdot \ln (1+k-p-q)
$$


A modicum of analysis shows us the above expression reaches its minimum if $k$ is chosen so that the following is true:

$$
\frac{v_{1}}{a}+\frac{v_{3}}{b}=\frac{v_{2}}{k}+\frac{v_{4}}{1+k-p-q}
$$

which is equivalent to (4).

One could be worried that such a $k$ might be unavailable for the agent, that is, that it could be negative or higher than either $p$ or $q$. Fortunately this never happens. Assume without loss of generalization that $p \leqslant q$. The derivative of (5) is a continuous function of $k$ in the segment $(0, p)$; with $k$ approaching 0 it approaches negative infinity, while with $k$ approaching $p$ it approaches positive infinity, therefore by the intermediate value theorem it reaches 0 at a point inside the segment $(0, p)$. At that particular point, as analysis of the second derivatives shows, (5) reaches a minimum. The number $k$ mandated by the logarithmic update method-and so by MIRE — is thus always available to the agent.

The cases with more worlds. We will consider what happens when we add more worlds to the proposition $A \cap \neg B$. In fact, the technique will, I hope, be evident after we deal with adding just a single world. To avoid much repetition I will skip the three remaining cases of the other logical combinations of $A$ and $B$.

As already mentioned, I believe it will be worthwile to start with the case in which just a single world is added to $A \cap \neg B$; that is, assume that $W=\left\{w_{0}, w_{1}, w_{2}\right.$, $\left.w_{3}, w_{4}\right\}, A$ is the proposition $\left\{w_{0}, w_{1}, w_{2}\right\}$, and $B$ is $\left\{w_{2}, w_{3}\right\}$. Label $b^{\prime}\left(\left\{v_{0}\right\}\right)$ with $a_{0}$ and $b^{\prime}\left(\left\{v_{1}\right\}\right)$ with $a_{1}$; using the equality $a_{0}=p-k-a_{1}$ we can write the expression to be minimized according to the local-logarithmic update method as

$$
-v_{0} \cdot \ln \left(p-k-a_{1}\right)-v_{1} \cdot \ln a_{1}-v_{2} \cdot \ln k-v_{3} \cdot \ln (q-k)-v_{4} \cdot \ln (1+k-p-q) .
$$

We will find expression (6)'s minimum when we calculate the values of $a_{1}$ and $k$ for which its partial derivatives w.r.t. those variables equal 0 (and second derivatives are positive, checking which we leave to the reader). Now, (6)'s partial derivative w.r.t $a_{1}$ equals 0 iff

$$
\frac{v_{1}}{a_{1}}=\frac{v_{0}}{p-k-a_{1}}
$$

from which by Observation 1 we get that

$$
\frac{v_{0}}{p-k-a_{1}}=\frac{v_{0}+v_{1}}{p-k} .
$$

Now, expression (6)'s partial derivative w.r.t. $k$ equals 0 precisely when

$$
\frac{v_{0}}{p-k-a_{1}}+\frac{v_{3}}{q-k}=\frac{v_{2}}{k}+\frac{v_{4}}{1+k-p-q},
$$

Ergo $\cdot$ vol. 3, no. $11 \cdot 2016$ 
which we now see to be equivalent to

$$
\frac{v_{0}+v_{1}}{p-k}+\frac{v_{3}}{q-k}=\frac{v_{2}}{k}+\frac{v_{4}}{1+k-p-q}
$$

that is, to (4), as required.

Notice now that if we add not just one, but more worlds to $A \cap \neg B$, nothing of essence changes. Instead of just two partial derivatives, we need to consider more, but the information we receive is again that for each world in $A \cap \neg B$, the ratio of the old credence in its singleton to the new credence in its singleton is the same, and so is also the same (by Observation 1) as the ratio of $b(A \cap \neg B)$ to $b^{\prime}(A \cap \neg B)$, at which point we turn to considering the partial derivative w.r.t. $k$ and get (4) immediately.

Adding now more worlds to the remaining three logical combinations of $A$ and $B$ changes, again, nothing of essence: we just need to consider more partial derivatives w.r.t. new variables, but what we learn is uniformly the information that inside the given logical combination of $A$ and $B$ the ratio of old credence to new credence is constant over singletons of all worlds, and (4) continues to hold regardless of how many (but finitely many) worlds we add. Q.E.D.

Proof of Fact 4

Fact 4 Let $A$ and $B$ be two propositions such that $A \cap B \neq \varnothing$ and let $b$ be the agent's prior belief function. Suppose that the agent's evidential constraints entail (only) her new credences $b^{\prime}(A)=p$ and $b^{\prime}(B)=q$. The belief function $b^{\prime}$ the agent should adopt according to MIRE is defined as follows:

For any $i \in\{1, \ldots, n\}, b^{\prime}\left(\left\{w_{i}\right\}\right)=\frac{b\left(\left\{w_{i}\right\}\right)}{-\lambda_{0}-\lambda_{1} \chi_{A, i}-\lambda_{2} \chi_{B, i}}$ with parameters $\lambda_{0}, \lambda_{1}$ and $\lambda_{2}$ determined by the following three equations:

$$
\begin{gathered}
\sum_{i=1}^{n} \frac{b\left(\left\{w_{i}\right\}\right)}{-\lambda_{0}-\lambda_{1} \chi_{A, i}-\lambda_{2} \chi_{B, i}}=1 ; \\
\sum_{i=1}^{n} \chi_{A, i} \frac{b\left(\left\{w_{i}\right\}\right)}{-\lambda_{0}-\lambda_{1} \chi_{A, i}-\lambda_{2} \chi_{B, i}}=p ; \\
\sum_{i=1}^{n} \chi_{B, i} \frac{b\left(\left\{w_{i}\right\}\right)}{-\lambda_{0}-\lambda_{1} \chi_{A, i}-\lambda_{2} \chi_{B, i}}=q .
\end{gathered}
$$

Proof: The constraint $b^{\prime}(A)=p$ is equivalent to $\sum_{i=1}^{n} \chi_{A, i} b^{\prime}\left(\left\{w_{i}\right\}\right)=p ; b^{\prime}(B)=q$ is equivalent to $\sum_{i=1}^{n} \chi_{B, i} b^{\prime}\left(\left\{w_{i}\right\}\right)=q$. Using the method of Lagrange multipliers (see, e.g., Cover \& Thomas 2012) we have that: 


$$
\begin{array}{r}
\Lambda=\sum_{i=1}^{n} b\left(\left\{w_{i}\right\}\right) \ln \left(\frac{b\left(\left\{w_{i}\right\}\right)}{b^{\prime}\left(\left\{w_{i}\right\}\right)}\right)-\lambda_{0}\left(\sum_{i=1}^{n} b^{\prime}\left(\left\{w_{i}\right\}\right)-1\right)-\lambda_{1}\left(\sum_{i=1}^{n} \chi_{A, i} b^{\prime}\left(\left\{w_{i}\right\}\right)-p\right)+ \\
-\lambda_{2}\left(\sum_{i=1}^{n} \chi_{B, i} b^{\prime}\left(\left\{w_{i}\right\}\right)-q\right) .
\end{array}
$$

Taking the partial derivative w.r.t. $b^{\prime}\left(\left\{w_{i}\right\}\right)$ we obtain

$$
\frac{\partial \Lambda}{\partial b^{\prime}\left(\left\{w_{i}\right\}\right)}=-\frac{b\left(\left\{w_{i}\right\}\right)}{b^{\prime}\left(\left\{w_{i}\right\}\right)}-\lambda_{0}-\lambda_{1} \chi_{A, i}-\lambda_{2} \chi_{B, i}
$$

This equals 0 precisely when $b^{\prime}\left(\left\{w_{i}\right\}\right)=\frac{b\left(\left\{w_{i}\right\}\right)}{-\lambda_{0}-\lambda_{1} \chi_{A, i}-\lambda_{2} \chi_{B, i}}$. Since the second derivatives are all positive, and our constraints define a closed and bounded set, we have arrived at a minimum. Q.E.D.

\section{Acknowledgments}

I would like to thank Juliusz Doboszewski, Richard Pettigrew, Tomasz Placek, and Jan Szwagierczak for commenting on various drafts of the paper, and Remco Heesen and Jan-Willem Romeijn for discussing some of the issues covered here with me. I presented portions and variants of the paper at various meetings of the Budapest-Kraków Research Group on Probability, Causality and Determinism, at Trends in Logic XIV (Ghent 2014), and the "Full and Partial Belief" workshop in Tilburg (2014); I'd like to thank all audiences for the comments. I am also grateful to the anonymous reviewers whose comments helped me to improve the text.

The research was aided by the joint project of the Polish and Hungarian Academies of Science "Probability, Causality and Determinism". It was also supported by the Sonata NCN grant 2015/17/D/HS1/o1912 "Epistemic inaccuracy and degrees of belief".

\section{References}

Bradley, Richard (2005). Radical Probabilism and Bayesian Conditioning. Philosophy of Science, 72(2), 342-364.

Cover, Thomas M. and Joy A. Thomas (2012). Elements of Information Theory. John Wiley \& Sons.

Diaconis, Persi and Sandy L. Zabell (1982). Updating Subjective Probability. Journal of the American Statistical Association, 77(380), 822-830.

Douven, Igor and Jan-Willem Romeijn (2011). A New Resolution of the Judy Benjamin Problem. Mind, 120(479), 637-670. 
Grove, Adam J. and Joseph Y. Halpern (1997). Probability Update: Conditioning vs. Cross-Entropy. In UAI'97 Proceedings of the Thirteenth conference on Uncertainty in artificial intelligence, 208-214.

Huisman, Leendert (2014). On Indeterminate Updating of Credences. Philosophy of Science, 81(4), 537-557.

Joyce, James M. (1998). A Nonpragmatic Vindication of Probabilism. Philosophy of Science, 65(4), 575-603.

Lange, Marc (2000). Is Jeffrey Conditionalization Defective by Virtue of Being Non-commutative? Remarks on the Sameness of Sensory Experiences. Synthese, 123(3), 393-403.

Leitgeb, Hannes (2014). The Stability Theory of Belief. Philosophical Review, $123(2), 131-171$.

Leitgeb, Hannes and Richard Pettigrew (2010a). An Objective Justification of Bayesianism I: Measuring Inaccuracy. Philosophy of Science, 77(2), 201-235.

Leitgeb, Hannes and Richard Pettigrew (2010b). An Objective Justification of Bayesianism II: The Consequences of Minimizing Inaccuracy. Philosophy of Science, 77(2), 236-272.

Levinstein, Benjamin A. (2012). Leitgeb and Pettigrew on Accuracy and Updating. Philosophy of Science, 79(1), 413-424.

Lukits, Stefan (2014). The Principle of Maximum Entropy and a Problem in Probability Kinematics. Synthese, 191(7), 1409-1431.

Osherson, Daniel (2002). Order Dependence and Jeffrey Conditionalization. Available at http://philpapers.org/rec/OSHODA.

Park, Ilho (2013). Simultaneous Belief Updates via Successive Jeffrey Conditionalization. Synthese, 190(16), 3511-3533.

Parry, Matthew, A. Philip Dawid, and Steffen Lauritzen (2012). Proper Local Scoring Rules. The Annals of Statistics, 4O(1), 561-592.

Pettigrew, Richard (2016). Accuracy and the Laws of Credence. Oxford University Press.

van Fraassen, Bas C. (1981). A Problem for Relative Information Minimizers in Probability Kinematics. British Journal for the Philosophy of Science, 32(4), 375379 .

Weisberg, Jonathan (2009). Commutativity or Holism? A Dilemma for Conditionalizers. British Journal for the Philosophy of Science, 6o(4), 793-812.

Williams, P. M. (1980). Bayesian Conditionalisation and the Principle of Minimum Information. British Journal for the Philosophy of Science, 31(2), 131144 . 